\title{
EUROPEAN CONSENSUS-BASED RECOMMENDATIONS FOR THE DIAGNOSIS AND TREATMENT OF KAWASAKI DISEASE - THE SHARE INITIATIVE
}

${ }^{*}$ Nienke de Graeff ${ }^{1},{ }^{*}$ Noortje Groot ${ }^{1,2,3}$, Seza Ozen ${ }^{4}$, Despina Eleftheriou ${ }^{5}$, Tadej Avcin ${ }^{6}$, Brigitte Bader-Meunier $^{7}$, Pavla Dolezalova ${ }^{8}$, Brian M. Feldman ${ }^{9}$, Isabelle Kone-Paut ${ }^{10}$, Pekka Lahdenne $^{11}$, Liza McCann ${ }^{3}$, Clarissa Pilkington ${ }^{5}$, Angelo Ravelli12 ${ }^{12}$ Annet van Royen-Kerkhof ${ }^{1}$, Yosef Uziel ${ }^{13}$, Bas Vastert ${ }^{1}$, Nico Wulffraat ${ }^{1}$, Sylvia Kamphuis ${ }^{2},{ }^{* *}$ Paul Brogan $_{2}{ }_{2}^{* *}{ }^{*}$ Michael W. Beresford ${ }^{3,14}$

1 Wilhelmina Children's Hospital, Utrecht, The Netherlands; 2 Sophia Children's Hospital, Erasmus University Medical Centre, Rotterdam, The Netherlands; ${ }^{3}$ Alder Hey Children's NHS Foundation Trust, Liverpool, United Kingdom; ${ }^{4}$ Dept. of Paediatric Rheumatology, Hacettepe University, Ankara, Turkey; ${ }^{5}$ Great Ormond Street Hospital for Children, London, United Kingdom; ${ }^{6}$ University Children's Hospital Ljubljana, Ljubljana, Slovenia; ${ }^{7}$ Necker Hospital, Assistance Publique-Hôpitaux de Paris, Paris, France; ${ }^{8}$ First Faculty of Medicine, Charles University and General University Hospital, Prague, Czech Republic; ${ }^{9}$ The Hospital for Sick Children, University of Toronto, Toronto, Canada; ${ }^{10}$ Bicêtre Hospital, APHP, university of Paris SUD, France; ${ }^{11}$ Hospital for Children and Adolescents, University of Helsinki, Helsinki, Finland; ${ }^{12}$ Gaslini Children's Hospital, Genoa, Italy; ${ }^{13}$ Meir Medical Centre, Sackler Faculty of Medicine, Tel Aviv University, Tel Aviv, Israel; ${ }^{14}$ Institute of Translational Medicine, University of Liverpool, Liverpool, United Kingdom

NB: $\quad$ * Joint first authors
$\quad * *$ Joint senior authors

\section{Corresponding author:}

Professor Michael W Beresford

Institute in the Park, University of Liverpool

Alder Hey Children's NHS Foundation Trust, Liverpool L14 5AB, United Kingdom

m.w.beresford@liverpool.ac.uk

+441512824536

\section{Key messages}

1. In children with Kawasaki disease, coronary artery aneurysms may be prevented by early institution of adequate anti-inflammatory therapy, typically intravenous immunoglobulin (IVIG).

2. Coronary artery outcomes associated with Kawasaki disease are currently worse than historically described, with diagnostic delay contributing to this. 
3. Patients resistant to IVIG are at highest risk of coronary artery aneurysms. However, there are no reliable biomarkers to identify such patients outside of Japan and thus stratify for adjunctive anti-inflammatory treatment

4. Meta analyses indicate a role for corticosteroids as adjunctive treatment to IVIG to prevent coronary artery aneurysms for high risk patients

5. We provide evidence-based recommendations for the diagnosis and treatment of Kawasaki disease in the light of these advances

\section{Key words}

Childhood / paediatric; Kawasaki disease; Systemic vasculitis; SHARE recommendations;

Treatment

\section{Disclosure Statement}

None declared

\section{Funding Statement}

This work was supported by the European Agency for Health and Consumers (EAHC; grant number 2011 1202) 


\section{ABSTRACT \\ Background}

The European SHARE initiative (Single Hub and Access point for paediatric Rheumatology in Europe) aimed to optimize care for children with rheumatic diseases. Kawasaki disease (KD) is the most common cause of acquired heart disease in children and an important cause of long-term cardiac disease into adulthood. Prompt diagnosis and treatment of KD is difficult due to the heterogeneity of the disease but is crucial for improving outcome. To date, there are no European internationally agreed, evidence-based guidelines concerning the diagnosis and treatment of KD in children. Accordingly, treatment regimens differ widely.

\section{Objectives}

To provide consensus based, European-wide evidence-informed recommendations for diagnosis and treatment of children with KD.

\section{Methods}

Recommendations were developed using the European League Against Rheumatism's standard operating procedures. An extensive systematic literature search was performed, and evidence-based recommendations were extrapolated from the included papers. These were evaluated by a panel of international experts via online surveys and subsequently discussed in three consensus meetings, using nominal group technique. Recommendations were accepted when $\geq 80 \%$ agreed.

\section{Results}

In total, 17 recommendations for diagnosis and 14 for treatment of KD in children were accepted. Diagnostic recommendations included laboratory and imaging workup for complete as well as incomplete KD. Treatment recommendations included the importance of early treatment in both complete and incomplete $\mathrm{KD}$, use of intravenous immunoglobulin, aspirin, corticosteroids for high-risk cases, and other treatment options for those with resistant disease.

\section{Conclusions}

The SHARE initiative provides international evidence-based recommendations for diagnosing and treating KD in children, facilitating improvement and uniformity of care. 


\section{INTRODUCTION}

Kawasaki disease $(K D)$ is the second most common systemic vasculitic illness of childhood after IgA vasculitis (IgAV, previously known as Henoch-Schönlein purpura)[1]. KD is more prevalent in Japanese children $(308 / 100,000)$ under the age of five years[2], a risk which is independent of geography.[3] In the UK, an indirect 2016 epidemiological survey indicated an incidence of 9.2/100,000 children under five years, with over-representation of Chinese and Japanese cases[4], but a recent direct British Paediatric Surveillance Unit (BPSU) epidemiological survey suggests an incidence of 4.55/100,000 children under 5 years (Tulloh and Brogan et al, manuscript submitted). In the USA, the incidence of KD is approximately 25/100,000 children under the age of five.[3]

Importantly, $\mathrm{KD}$ is the most common cause of acquired heart disease in children in developed countries, causing coronary artery aneurysms (CAA) in up to $25 \%$ of untreated patients due to coronary vasculitis. This declines to approximately $4 \%$ with intravenous immunoglobulin (IVIG) treatment.[3, 5] Mortality varies by population: $0.015 \%$ in Japan[3]; $0.17 \%$ in the USA[3] and $0.36 \%$ in the UK.[4] KD remains an important cause of long-term cardiac disease into adulthood. $[3,6,7]$ The complexity and heterogeneity of presentation of KD, broad differential diagnosis, and lack of a diagnostic test can be important barriers for making a prompt diagnosis.

In 2012, the European initiative SHARE (Single Hub and Access point for paediatric Rheumatology in Europe) was launched to optimize care for children and young adults with Paediatric Rheumatic Diseases (PRD).[8] To date, SHARE-recommendations for paediatric antiphospholipid syndrome, juvenile dermatomyositis, familial Mediterranean fever/autoinflammatory diseases and childhood-onset lupus have been published.[9-13] Although the American Heart Association (AHA) provided updated and detailed guidelines for KD in 2017[3], there are no internationally-agreed, evidence-based recommendations for KD in children. Treatment regimens still differ widely between centres, and internationally.[5, 14] Thus, the SHARE recommendations aim to fulfil this important unmet need to provide a practical tool for optimal care of children with KD.

\section{METHODS}

A panel of 17 experts in paediatric rheumatology and systemic vasculitides from across Europe was established to develop evidence-based recommendations for diagnosing and treating childhood KD. Experts needed to be senior consultants with at least 10 years' experience working in a major tertiary paediatric rheumatology referral centre routinely looking after children with KD and as part of a multi-disciplinary team. As SHARE was a European Union (EU)-funded project, only experts from across Europe were able to be selected, representing a balance between experience and geography, although the panel carefully considered literature and other published recommendations from experts from across the globe. The panel were informed by expert recommendations from paediatric cardiology and infectious diseases and 
other specialists, but due to the specific scope of the SHARE initiative, the panel did not include directly experts from these specialties in the process. The panel used the previously described[12] SHARE methods and following the European League Against Rheumatism (EULAR) standardised operating procedure for developing best practice recommendations.[15]

\section{Systematic literature review and study selection}

Based on specific research questions, the PubMed/MEDLINE, EMBASE and Cochrane databases were systematically searched on $20^{\text {th }}$ June 2013 resulting in a set of articles that were then assessed (Supplementary Figure S1). All systemic vasculitides synonyms were searched using $\mathrm{MeSH} / \mathrm{Em}$ tree terms, title and abstract, using a validated filter pertaining to children and adolescents only[16] (Supplementary Table S1). Articles were assessed using pre-specified inclusion/exclusion criteria (Supplementary Table S2). The comprehensive literature review was undertaken inclusive of these other forms of systemic vasculitis to ensure no manuscripts including data on KD along with any of these other forms were missed. All articles were screened independently by two reviewers (NdG, NG) and full text assessed when necessary to determine eligibility. Disagreement was resolved by a third reviewer (MWB); agreement was reached in all cases. This literature review was cross-referenced with key articles that had informed a contemporaneous UK national guideline for KD[5].

Additional key KD articles identified between the initial literature search and the final manuscript drafting (May 2018) were identified using the same search strategy. Whilst these latter did not directly inform the recommendations, they were included in the manuscript commentary to provide up-to-date face validity and contextualisation, particularly to incorporate updated AHA 2017 guidance.[3]

\section{Validity assessment}

Papers pertaining to KD were analysed using standardized data extraction and scoring forms by two experts (PB and $\mathrm{DE}$ ); any discrepancies were resolved by a third expert (MWB). Data were extracted using predefined scoring forms for demographics, diagnostic[17] and therapeutic[18] studies. Adapted classification tables for diagnostic[19] and therapeutic[20] studies were used to determine the level of evidence and strength of each recommendation (Supplementary Tables S3, S4).

\section{Establishment of recommendations}

Provisional statements regarding diagnosis and treatment were developed using data from included articles (NdG, NG, SO, SK, PB and MWB). These statements were presented to the expert committee ( $n=14 / 17$ of the experts) in an online survey (100\% response rate). Recommendations were revised according to responses and discussed at three face-to-face consensus meetings in March 2014 (Genoa, n=14/17 expert participants); January 2015 (Utrecht, $n=10 / 17$ experts) and March 2015 (Barcelona, $n=16 / 17$ experts). Nominal group 
technique was used to reach consensus[21], with final recommendations accepted with $\geq 80 \%$ agreement.

\section{RESULTS}

\section{Literature search and formulation of recommendations}

The literature search yielded 826 articles relating to KD (Figure S1). References concerning rare paediatric systemic vasculitides and IgA vasculitis informed additional recommendations described in separate manuscripts (Figure S1). A total of 31 recommendations were accepted with $100 \%$ agreement: 17 relating to diagnosis and 14 concerning treatment of KD.

\section{Diagnosis of Kawasaki Disease}

Table 1 summarises the SHARE recommendations for diagnosing KD and incomplete KD. 


\section{Table 1: SHARE Recommendations for the diagnosis and assessment of KD}

\section{KD Recommendations - Diagnosis}

1. There are different diagnostic criteria for KD. The AHA diagnostic criteria should be used to diagnose complete KD (see Table 2).

2. The diagnosis of KD should be considered in any child with a febrile exanthematous illness and evidence of inflammation, particularly if it persists longer than 4 days.

3. KD diagnosis and treatment should not be delayed if:

- $\quad 5 / 6$ diagnostic criteria of KD are present before day 5 of fever.

- $\quad$ Coronary Artery Aneurysms (CAA) or coronary dilatation are present.

- $\quad$ There is evidence of persistent ( $\geq 5$ days) elevation of inflammatory markers and/or persistent fever, especially in infants or younger children without other explanation.

4. In a patient in whom KD is suspected, but all criteria have not yet been fulfilled, the following clinical signs strengthen the suspicion of KD: Irritability

- New erythema and/or induration at the site of previous BCG immunisation.

5. It is recognized that there are incomplete cases of KD (who do not fulfil the AHA-criteria); however, these patients may still be at risk of CAA, particularly infants.

6. Criteria should be designed and validated to help diagnose incomplete KD*.

7. In children presenting with less than 5 out of 6 criteria for KD ('incomplete KD'), with evidence of unexplained systemic inflammation (e.g. elevated CRP, ESR or WBC), an echocardiogram should be considered.

8. The following laboratory values should be determined: ESR, CRP, full blood count and liver function (bilirubin, AST/ALT), albumin, natremia, renal function and urinalysis. Ferritin and fibrinogen should be considered if there is a concern for macrophage activation syndrome.

9. Cerebrospinal fluid analysis may be important to rule out infectious meningitis.

10. The following laboratory values can be important in assessing risk stratification for IVIG resistance: Low sodium, raised bilirubin, raised ALT, low platelet count, high CRP, low albumin**.

11. The following laboratory values can be important in monitoring inflammation: ESR (prior to IVIG), CRP and full blood count.

12. All patients with suspected KD should undergo echocardiography and ECG at baseline, as soon as the diagnosis is suspected.

13. An intermediate echocardiogram, 2 weeks after the first IVIG, should be performed in all patients with KD whose initial echo was normal and in whom disease activity has been arrested.

14. All patients with KD should undergo echocardiography at 6-8 weeks after disease onset.

15. In those with ongoing active inflammation (increasing or persistently elevated CRP and/or persisting signs and symptoms), ECG and echocardiography should be performed at least weekly to monitor the possible development of cardiac sequelae.

16. In those with coronary abnormalities detected on initial echocardiography, echocardiography should then be performed at least weekly to monitor progression until there is clinical stabilization.

17. In children with CAA, ECG and echocardiography should be performed 3 to 6 -monthly, depending on the severity of the CAA***

\begin{tabular}{|c|c|}
\hline LoE & SoR \\
\hline 4 & $\mathrm{D}$ \\
\hline 4 & $\mathrm{D}$ \\
\hline 4 & D \\
\hline 4 & $\mathrm{D}$ \\
\hline 4 & $D$ \\
\hline 4 & $D$ \\
\hline 3 & $\mathrm{D}$ \\
\hline 3 & $\mathrm{C}$ \\
\hline 4 & D \\
\hline 3 & C \\
\hline $2 \mathrm{~B}$ & $\mathrm{C}$ \\
\hline $1 \mathrm{~A}$ & $B$ \\
\hline $1 \mathrm{~A}$ & $B$ \\
\hline $2 \mathrm{~A}$ & C \\
\hline $2 A$ & C \\
\hline $2 A$ & $C$ \\
\hline 4 & D \\
\hline
\end{tabular}


Footnotes:

*These SHARE recommendations were formulated prior to publication of the American Heart Association (AHA) 2017 recommendations $^{3}$, which describe an algorithm for the diagnosis and treatment of incomplete KD cases. Although the AHA algorithm is not evidence-based, it provides a useful diagnostic framework (see main text).

** See also Supplementary Table S5 which provides details of the 3 main scoring systems used to determine risk of IVIG resistance.

${ }^{* * *}$ Or as otherwise recommended by an expert paediatric cardiologis

Abbreviations:

AHA, American Heart Association; ALT, alanine transaminase; AST, aspartate transaminase; CAA, coronary artery aneurysm; CRP, C-reactive protein; ECG, electrocardiogram; ESR, erythrocyte sedimentation rate; IVIG, intravenous immunoglobulin; KD, Kawasaki disease; WBC, white blood cell count

LoE, level of evidence: $1 \mathrm{~A}$, meta-analysis of cohort studies; $1 \mathrm{~B}$, meta-analysis of case control studies; $2 \mathrm{~A}$, cohort studies; $2 \mathrm{~B}$, case control studies; 3 , non-comparative descriptive study; 4 expert opinion. SoR, strength of recommendation; $A$, based on level 1 evidence; $B$, based on level 2 or extrapolated from level 1; $C$, based on level 3 or extrapolated from level 1 or $2 ; D$, based on level 4 or extrapolated from level 3 or 4 expert opinion.

\section{Table 2: Kawasaki disease: Diagnostic criteria (AHA 2017³)}

\begin{tabular}{ll}
\hline Criteria & Description \\
\hline Fever & Duration of 5 days or more PLUS 4 of 5 of the following: \\
1. Conjunctivitis & Bilateral, bulbar, conjunctival injection without exudate \\
2. Lymphadenopathy & Cervical, often $>1.5 \mathrm{~cm}$ usually unilateral \\
3. Rash & Rash: Maculopapular, diffuse erythroderma or erythema multiforme \\
4. Changes of lips or oral mucosa & Red cracked lips; "strawberry" tongue; or diffuse erythema of oropharynx \\
5. Changes to extremities & Erythema and oedema of palms and soles in acute phase and periungal \\
& desquamation in subacute phase \\
\hline
\end{tabular}

NB. KD may be diagnosed with fewer than 4 of these features if coronary artery abnormalities are detected (Figure 1). 


\section{Diagnostic criteria for KD}

There is no diagnostic test for KD; thus, diagnosis rests on clinical criteria and laboratory findings. To establish the diagnosis according to the Diagnostic Guidelines of the Japan KD Research Committee, any five of the six criteria in Table 2 must be present.[5] The AHA (2004) diagnostic criteria are similar, except that fever is mandatory, and four of the remaining five criteria are required.[14] The expert panel assessed the merits and strengths of each, and recommended that the AHA diagnostic criteria should be used for complete KD. Subsequently, the AHA revised their diagnostic criteria, as summarised in Table 2 . These are broadly similar to the 2004 criteria, but now acknowledge that diagnosis may be made earlier than day 5 of fever, if fever plus $\geq 4$ principle clinical features are present, in line with the SHARE recommendations. However, many patients have some but not all of the clinical features of KD and may still be at risk of CAA (see below). Clinical features may present sequentially, such that an 'incomplete' case can evolve into a 'complete' case.[14] Thus, the diagnosis of KD must be considered in any child with a febrile exanthematous illness and evidence of inflammation, particularly if it persists longer than 4 days. $[5,14,22]$

\section{Diagnosing KD before day 5 of fever}

The SHARE experts acknowledged that the requirement for fever $\geq 5$ days may lead to delayed treatment. While fever duration has historically been of importance for the standardisation of case definitions, clinicians should not delay diagnosing KD and instituting treatment if 5 out of 6 diagnostic criteria of KD are present before day 5 of fever, or if CAA (Z-score $\geq 2.5$ ) or coronary dilatation $(Z$-score $>2$, but $<2.5)$ are present.[5, 14, 22]

\section{Diagnosing Incomplete Kawasaki disease}

Whilst the diagnosis of KD is generally straightforward in patients fulfilling all the criteria for KD ('complete' KD), many patients have only some of the clinical features.[3, 5, 14, 22] However, they may still be at risk of CAA, especially infants who may have prolonged fever alone or fleeting clinical signs. The panel acknowledged that diagnosing KD in patients with incomplete clinical criteria relies on a high index of suspicion, in agreement with other current guidelines. [3, $5,14]$ In these situations, early echocardiography is recommended. This may reveal evidence of coronary vasculitis, confirming the diagnosis of KD. Notably, however, a negative echocardiogram does not exclude the diagnosis of KD.

The definition of 'incomplete KD' can cause confusion. As is the case for complete KD, the requirement of fever $\geq 5$ days to fulfill current diagnostic criteria may delay treatment unnecessarily. The expert panel recommended that an important research priority is to design and prospectively validate criteria to help diagnose incomplete KD in children. The AHA 2017 recommendations provide some guidance in this respect, defining incomplete $K D$ as children with fever $\geq 5$ days and 2 or 3 clinical criteria, or infants with fever for $\geq 7$ days without other explanation.[3] Thereafter, treatment decisions are determined by laboratory tests including inflammatory markers (Figure 1). These AHA recommendations offer a practical solution for the 
diagnosis and treatment of incomplete KD cases, but are not evidence-based, as acknowledged by McCrindle et al.[3]

Figure 1

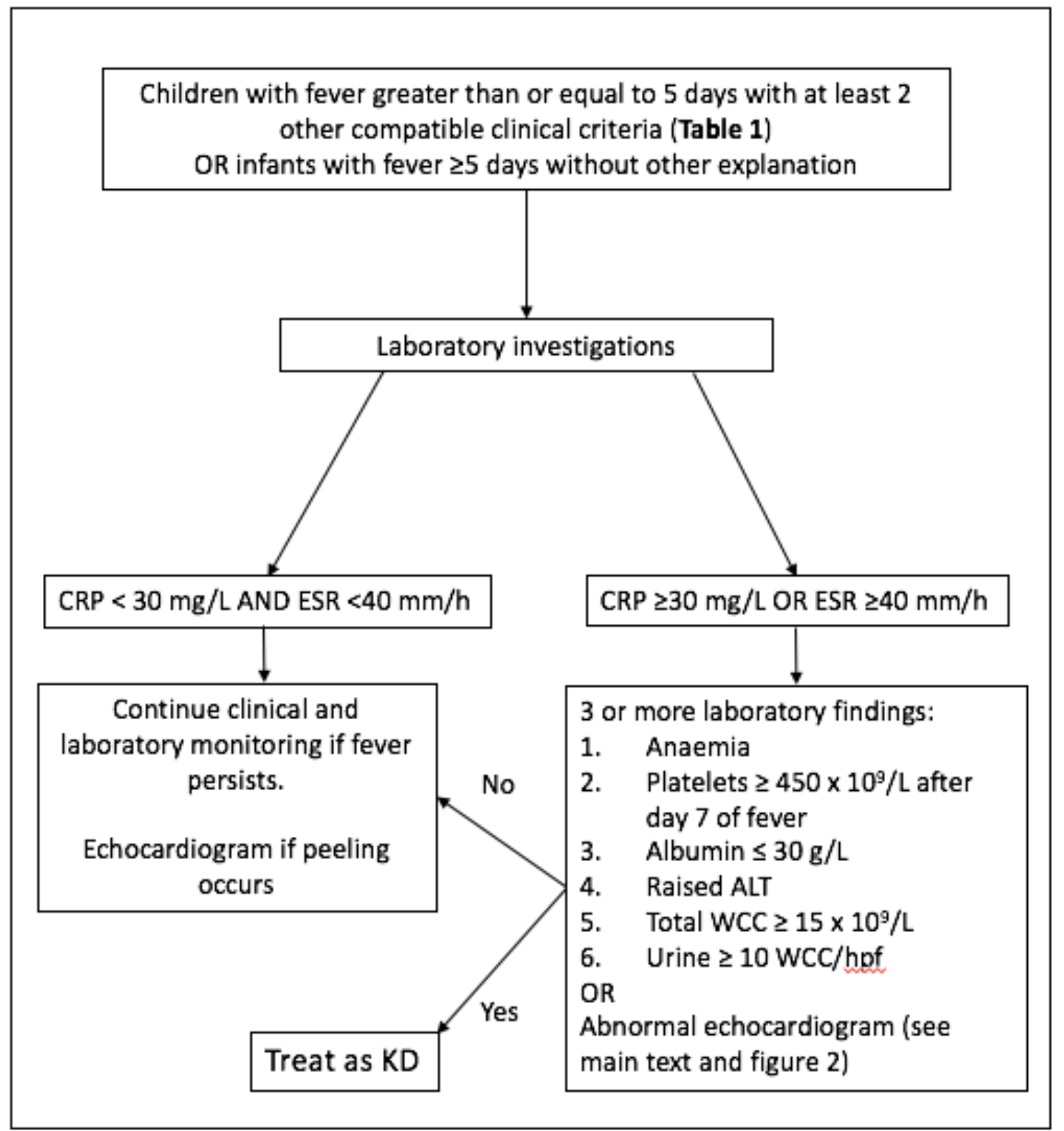

Figure 1: Management of suspected incomplete Kawasaki disease KD (adapted from AHA 2017[3]). $C R P=C$-reactive protein; $E S R=$ erythrocyte sedimentation rate; $W C C=$ white cell count; $A L T=a l a n i n e ~ t r a n s a m i n a s e ; ~ h p f=h i g h$ power field. 


\section{Laboratory work-up of suspected Kawasaki Disease}

The diagnosis of KD is unlikely in the absence of significant systemic inflammation. Certain laboratory parameters may help stratify the severity of KD and thus help inform therapeutic decisions. Therefore, erythrocyte sedimentation rate (ESR), C-reactive protein (CRP), full blood count, electrolytes, renal and liver function (including bilirubin, AST or ALT, and albumin) should be monitored in all patients.[3, 5, 14] Notably, ESR is only useful prior to IVIG therapy, since this may be elevated post-IVIG as a consequence of binding to red blood cells. [23, 24] It is equally important to rule out severe infections (such as meningitis), and/or identify other systemic inflammatory diseases or complications of KD including macrophage activation syndrome (also referred to as secondary haemophagocytic lymphohistiocytosis, HLH). $[3,5]$ Thus, consideration of a full septic screen (including consideration of lumbar puncture) and serum ferritin are recommended. Evidence of infection might occur in patients with KD, and should not deter clinicians from treating both entities[25].

\section{Scoring systems to predict high-risk cases}

Several scoring systems have been developed to identify children at highest risk of IVIG resistance and hence highest risk of developing CAA[26-28]. Kobayashi et al. developed a model to predict unresponsiveness to IVIG in Japanese children with KD based on a cut-off point $\geq 4$ calculated as per below: sodium $\leq 133 \mathrm{mmol} / \mathrm{L}$ ( 2 points); days of illness at initial treatment $\leq 4$ ( 2 points); aspartate aminotransferase $\geq 100 \mathrm{IU} / \mathrm{L}$ (2 points); percentage of neutrophils $\geq 80 \%$ ( 2 points); C-reactive protein $\geq 100 \mathrm{mg} / \mathrm{L}$ ( 1 point); age $\leq 12$ months ( 1 point); platelet count $\leq 300 \times 10$ 9/L (1 point)[27] (see Supplementary Table S5). This model was used to define severe cases in a pivotal clinical trial of corticosteroids, because IVIG resistance is known to be a strong risk factor for the development of CAA[27]. The Kobayashi, Egami, and Sano scores (see Supplementary Table S5), when tested in North American patients, demonstrated comparably high specificity for predicting IVIG non-response, but with relatively low sensitivity[29]. These data suggested that in non-Japanese children, a positive Kobayashi score might identify a patient at high risk of IVIG resistance, but a negative score may not reliably exclude a high-risk case. More recently, studies from the UK and Germany also demonstrated suboptimal performance of the Kobayashi score for predicting high-risk cases[30, 31]. Therefore, clinicians must adopt a pragmatic approach and synthesise an overall picture of disease severity based on clinical features and laboratory parameters. Factors that increase risk include young age (i.e. $<12$ months), low serum sodium, high ALT, low albumin, high bilirubin, high CRP, low platelet count, falling haemoglobin, features of $\mathrm{HLH}$ and shock, and are all important features to consider when assessing risk and hence choice of primary KD treatment modality.

\section{Echocardiography and monitoring systemic inflammation}

As up to $25 \%$ of untreated KD cases develop CAA, and a significant proportion have other cardiac manifestations, including pericardial effusion, pericarditis, myocarditis, valvular 
incompetence, cardiac failure and even myocardial infarction, all patients with suspected KD should undergo echocardiography at baseline, as soon as it is suspected.[3, 5, 14, 22] Treatment should not be delayed whilst awaiting echocardiography.[3, 5, 14, 22] In view of the potential rapidly evolving nature of this critical complication, all patients with KD should have an intermediate echocardiogram, two weeks after administration of the first IVIG, including those whose initial echo was normal and in whom disease activity has been arrested.[3, 5, 14, 22, 32-35] All patients should undergo echocardiography at 6-8 weeks after disease onset.[3, 5] Although not noted as a specific recommendation, it was acknowledged that all patients should have echocardiography undertaken by a paediatric cardiologist or by echocardiographers trained specifically in paediatric cardiology working directly within a paediatric cardiology team.

Historically, resolution of fever has been used as a metric of therapeutic outcome success in KD. However, some patients may become afebrile but still have significant ongoing systemic inflammation as indicated by elevation of acute phase reactants, including CRP. Indeed, recent clinical trials have employed resolution of fever and normalisation of CRP in their therapeutic design[36], emphasising that temperature alone should not be used to gauge the degree of systemic inflammation, as reflected in recent clinical guidelines.[3, 5] Close monitoring of patients with increasing or persistently elevated CRP and/or persisting signs of systemic inflammation is therefore critical, combined with regular cardiology reviews including at least fortnightly ECG and echocardiography to assess cardiac sequelae.[3, 5] Erythrocyte sedimentation rate should not be taken into account after IVIG (as an elevation of proteinemia leads to an elevation of ESR). In those with coronary abnormalities including CAA, at least weekly echocardiography should be considered to monitor progression until clinical stabilization. Among those with CAA, ECG and echocardiography should be performed every 3 to 6 months (or as specified by a paediatric cardiologist for individual cases), depending on CAA severity.[3, 5] A final important caveat in relation to echocardiography for young children who present with systemic inflammation, is that other inflammatory diseases might be associated with transient coronary artery dilatation, particularly systemic-onset juvenile idiopathic arthritis.[37]

\section{Treating Kawasaki Disease}

Table 3 summarises the SHARE recommendations for treating KD in childhood.

\section{IVIG}

Randomized controlled trials and meta-analyses have demonstrated unequivocally, that early recognition and treatment of KD with IVIG and aspirin reduces the occurrence of CAA.[38, 39] Therefore, the panel recommended strongly that IVIG and aspirin should be started as soon as a patient is diagnosed with complete or incomplete $\mathrm{KD}$. In keeping with previous guidance[3, 5], treatment should include a dose of $2 \mathrm{~g} / \mathrm{kg}$ IVIG as a single infusion, in view of greater 
therapeutic effect in preventing CAA when compared to a lower, divided dose regimen.[40] As the Kobayashi criteria in non-Japanese patients may not reliably exclude IVIG resistance even if 'negative' [29], close monitoring of patients is critical, taking into account temperature, acute phase reactants (particularly CRP post-IVIG), clinical symptoms and signs of systemic inflammation. 
1. As soon as a patient is diagnosed with KD, treatment should be initiated*. This applies to both complete and incomplete KD.

2. Treatment of KD should include IVIG at a dose of $2 \mathrm{~g} / \mathrm{kg}$ as a single infusion.

3. In non-Japanese patients, the Kobayashi criteria may indicate risk of IVIG resistance if 'positive' (score $\geq 4$ ) but may not reliably exclude IVIG resistance if 'negative' (score<4).

4. All patients diagnosed with KD who are treated with IVIG should be treated with aspirin at a dose of 30-50mg/kg/day until fever has settled for $48 \mathrm{hrs}$; clinical features are improving; and CRP levels are falling.

5. The dose of aspirin should subsequently be reduced to an antiplatelet dose of 3-5mg/kg once daily when fever and inflammation have subsided.

6. If aneurysms persist in the convalescent phase of $\mathrm{KD}$, antiplatelet therapy in the form of low-dose aspirin (3-5mg/kg) should be continued long-term, at least until the aneurysms resolve.

7. In patients with CAA that resolve, long term aspirin (3-5mg/kg/day) should be considered, taking into account the risk-benefit ratio for individual patients.

8. Corticosteroid treatment should be given to patients with severe $\mathrm{KD}^{*}$ :

a. Who are IVIG resistant, that is, with ongoing fever and/or persistent inflammation or clinical signs $\geq 48 \mathrm{~h}$ after receiving IVIG as a single dose of $2 \mathrm{~g} / \mathrm{kg}$. A second dose of IVIG is at the discretion of the treating physician.

b. Kobayashi score $\geq 5$ (see Supplementary Table S5)

c. With features of haemophagocytic lymphohistiocytosis $(\mathrm{HLH})$

d. With features of shock

e. Who are under the age of 1 year

f. Who present with coronary and/or peripheral aneurysms

9. If corticosteroids are indicated, the following regimens would be reasonable:

- $\quad$ Regimen 1: methylprednisolone $0.8 \mathrm{mg} / \mathrm{kg} \mathrm{BD} \mathrm{IV} \mathrm{for} \mathrm{5-7} \mathrm{days} \mathrm{or} \mathrm{until} \mathrm{CRP} \mathrm{normalizes;} \mathrm{then} \mathrm{convert} \mathrm{to} \mathrm{oral}$

prednisone/prednisolone $2 \mathrm{mg} / \mathrm{kg} / \mathrm{day}$ and wean off over next 2-3 weeks.

- $\quad$ Regimen 2: methylprednisolone $10-30 \mathrm{mg} / \mathrm{kg}$ (up to maximum of $1 \mathrm{~g} / \mathrm{day}$ ) once daily for 3 days followed by oral

prednisone/prednisolone $2 \mathrm{mg} / \mathrm{kg}$ per day until day 7 or until CRP normalizes; then wean over next 2-3 weeks.

10. TNF-alpha blockade (e.g. infliximab) should be considered in KD patients with persistent inflammation despite IVIG, aspirin and corticosteroid treatment, after consultation with a specialist unit.

11. The use of DMARDs such as ciclosporin, cyclophosphamide, and methotrexate, along with anakinra and plasma exchange, cannot be recommended, except on an individual basis after consultation with a specialist unit.

12. In the presence of giant aneurysms (internal diameter $\geq 8 \mathrm{~mm}$; or Z-score $\geq 10$; and/or coronary artery stenosis) warfarin should be administered (in addition to aspirin), after initial heparinisation; heparin can be stopped when a stable INR between 2-3 is reached.

\begin{tabular}{|c|c|}
\hline LoE & SoR \\
\hline $1 \mathrm{~A}$ & $A$ \\
\hline $1 \mathrm{~A}$ & $A$ \\
\hline $2 \mathrm{~A}$ & $\mathrm{C}$ \\
\hline $2 \mathrm{~A}$ & $\mathrm{C}$ \\
\hline 3 & $D$ \\
\hline 3 & $D$ \\
\hline 4 & $D$ \\
\hline$* * 1 \mathrm{~A}$ & $A$ \\
\hline $1 \mathrm{~A}$ & C \\
\hline 3 & C \\
\hline 4 & $D$ \\
\hline 4 & $D$ \\
\hline 4 & $D$ \\
\hline 4 & $D$ \\
\hline $2 \mathrm{~A}$ & $B$ \\
\hline $2 \mathrm{~A}$ & $\mathrm{C}$ \\
\hline 4 & $\mathrm{D}$ \\
\hline $2 \mathrm{~B}$ & $\mathrm{C}$ \\
\hline
\end{tabular}


13. If symptoms of ischaemia or obstruction occur in a patient with $\mathrm{KD}$, a paediatric cardiologist/cardiac surgeon/interventional radiologist (depending on local expertise available) should be consulted immediately

14. Immunisation with all vaccines should be deferred for at least 6 months following an episode of KD treated with IVIG.

Abbreviations and footnotes:

* Treatment should not be delayed whilst awaiting echocardiography.

** Level of evidence $1 \mathrm{~A}$ and strength of recommendation $\mathrm{A}$ for this overall statement in relation to severe $\mathrm{KD}$.

Abbreviations: C-Reactive Protein (CRP); Coronary Artery Aneurysms (CAA); Disease Modifying Anti-Rheumatic drugs (DMARDs); haemophagocytic lymphohistiocytosis (HLH); Intravenous Immunoglobulin (IVIG); Kawasaki disease (KD):

LoE, level of evidence: $1 \mathrm{~A}$, meta-analysis of randomised controlled trials; $1 \mathrm{~B}$, randomised controlled study; $2 \mathrm{~A}$, controlled study without randomisation; $2 \mathrm{~B}$, quasi-experimental study; 3 , descriptive study; 4 expert opinion; SoR, strength of recommendation: A, based on level 1 evidence; B, based on level 2 or extrapolated from level 1; C, based on level 3 or extrapolated from level 1 or 2; D, based on level 4 or extrapolated from level 3 or 4 expert opinion. 


\section{Aspirin}

All patients should initially receive aspirin at a dose of $30-50 \mathrm{mg} / \mathrm{kg} /$ day, in 3-4 divided doses. Meta-analysis comparing the $30-50 \mathrm{mg} / \mathrm{kg} /$ day dose with high-dose $(80-120 \mathrm{mg} / \mathrm{kg} / \mathrm{day})$, both combined with IVIG, demonstrated no significant difference in the incidence of CAA.[41] Aspirin should be reduced to an antiplatelet dose of 3-5 mg/kg/day, but only after the fever has settled for 48 hours, clinical features are improving, and CRP levels are falling.[3,5] Aspirin can be stopped if the echocardiogram at 6-8 weeks remains normal. If CAA persist in the convalescent phase, continuation of low-dose aspirin $(3-5 \mathrm{mg} / \mathrm{kg} /$ day) is recommended long-term, at least until the aneurysms resolve.[3, 5] In patients with resolved CAA, long term aspirin (3-5 $\mathrm{mg} / \mathrm{kg} /$ day) should still be considered, taking into account the risk-benefit ratio for individual patients. It is increasingly recognized that patients with regressed aneurysms may demonstrate coronary artery endothelial function abnormalities comparable to those with persistent CAA.[6] The adverse effects of late KD vasculopathy, even in those with resolved CAA, is increasingly recognized.[3,5] It should be remembered that ibuprofen and other nonsteroidal antiinflammatory drugs interfere with the antiplatelet effect of aspirin and thus should be avoided if possible; a point emphasized in the recent AHA guidelines.[3] It is possible that future guidance may recommend low dose aspirin (3-5 mg/kg/day) at all stages of $K D$, as suggested by data from a retrospective cohort[42]; whilst the SHARE group acknowledged this recent development, there has never been a prospective controlled clinical trial to support this approach.

\section{Treatment with Corticosteroids}

Up to $20-40 \%$ of patients are IVIG resistant, and are at increased risk of developing CAA.[36, 43-45] In the UK, CAA rates remain significantly higher (19-29\%) despite IVIG, a worrisome complication rate that has remained stable over 25 years of surveillance.[4, 46] Similarly poor outcomes are now recognized in Sweden, and Russia.[47, 48] Although therapeutic delay is a major contributing factor to these poor outcomes, some authors speculate that IVIG response may vary across populations[46], perhaps due to genetic differences such as Fc gammareceptor polymorphisms[3], emphasizing the need for adjunctive primary treatment in some patients.

Whilst significant equipoise remains regarding the use of corticosteroids for unselected KD patients, the use of corticosteroids as primary adjunctive treatment of patients with severe $K D[49]$, has an increasingly compelling evidence-base.[36, 43, 44, 50-53] Meta-analysis of 16 comparative studies involving $2746 \mathrm{KD}$ patients demonstrated that early addition of corticosteroids to conventional IVIG therapy is associated with reduced risk of CAA compared with IVIG therapy alone (odds ratio $0.424 ; 95 \% \mathrm{Cl}, 0.270-0.665$ ).[44] This beneficial effect was only observed when corticosteroids were used as primary therapy rather than rescue therapy for IVIG resistance. It was most beneficial for patients who were determined at baseline to have high-risk for IVIG resistance. The authors highlighted the importance of prompt diagnosis and 
treatment: meta-regression analyses demonstrated that the overall efficacy of corticosteroids was negatively correlated with illness duration before corticosteroid therapy.[44] Thus, the need for more robust clinical risk scoring systems to identify high-risk patients is underlined.

Notwithstanding the ongoing debate regarding the definition of KD severity in non-Japanese patients, it is unclear whether corticosteroids benefit patients with less severe $K D[3,5]$, and the optimal corticosteroid dosing regimen to use is uncertain. $[5,49]$ Thus, preventing potentially life-long cardiac sequelae needs to be balanced against careful vigilance for corticosteroidrelated complications. In view of this, the expert panel recommended that adjunctive primary corticosteroid treatment should be given to patients: a) who are IVIG resistant, with ongoing fever and/or persistent inflammation or clinical signs $\geq 48$ hours after receiving a single dose of IVIG[5] (a second dose of IVIG alongside corticosteroids is at the discretion of the treating physician[5]); b) have a Kobayashi score $\geq 4$ [29]; c) have features of HLH[54]; and/or d) have features of shock. $[55,56]$ The panel defined additional 'high-risk groups' who are also likely to benefit from primary adjunctive corticosteroids, namely: infants $<1$ year of age[5], and in patients who present with coronary and/or peripheral aneurysms at diagnosis[5], although this may impact on damage limitation only.[44] As the meta-analysis of corticosteroid treatment in $K D$ does not provide definite evidence for optimal treatment regimens[43, 44, 49], treating clinicians will need to determine the corticosteroid regimen for individual patients. If corticosteroids are indicated, the panel noted two treatment regimens that in their consensus opinion would be reasonable (Table 3).

\section{TNF-alpha blockade and other treatments}

An increasing number of studies have explored the potential use of TNF-alpha blockade in children with IVIG-resistant KD.[57-61] Although these studies have demonstrated no significant differences in cardiac-related outcomes, such as reduction in CAA, they generally demonstrate a significant impact on reducing the acute-phase response, fever, and potentially length of hospital stay. Therefore, use of anti-TNF-alpha medication (e.g. infliximab) was recommended for consideration in KD patients with persistent inflammation despite IVIG, aspirin and corticosteroid treatment; albeit after consultation with a specialist unit. The panel did not make specify dosing or need for additional doses of anti-TNF-alpha medication, which were felt to be beyond the scope of these recommendations. Evidence supporting the use of other treatments such as ciclosporin, cyclophosphamide, methotrexate, along with anakinra or plasma exchange, was less robust and therefore could not be routinely recommended, apart from on a case-by-case basis after consultation with a specialist unit.

\section{Anti-coagulation and anti-aggregation}

Those with medium sized aneurysms ( $Z$ score $5-10$ ) should also take aspirin at $3-5 \mathrm{mg} / \mathrm{kg} / \mathrm{day}$, and consider addition of an antagonist of ADP-mediated activation platelet aggregation such as Clopidogrel, at a dose of $0.5-1 \mathrm{mg} / \mathrm{kg} / \mathrm{daily}$.[3] Managing patients with giant aneurysms 
(internal diameter $\geq 8 \mathrm{~mm}$; or Z-score $\geq 10$; and/or coronary artery stenoses) includes anticoagulation as well as antiplatelet therapy with aspirin.[62] Warfarin should be administered in addition to aspirin after initial heparinisation, and heparin can be stopped when a stable INR of $2-3$ is reached.[22, 62, 63] If symptoms of ischaemia or vascular occlusion occur in a patient with $\mathrm{KD}$, a paediatric cardiologist/cardiac surgeon/interventional radiologist (depending on local expertise available) should be consulted, if not already closely involved in management.[3,5] Detailed advice on the use of low molecular weight heparin, clopidogrel and other thienopyridines, thrombolysis and other acute revascularisation procedures was not considered in the SHARE process, but these issues have been addressed in the recent AHA guidance, albeit with limited evidence to inform guidance.[3]

\section{Immunisation}

Immunisation with all live vaccines should be deferred for at least 6 months following an episode of KD treated with IVIG, mainly due to the potential lack of effectiveness following IVIG[64, 65]. Thereafter, all vaccines should be administered as recommended by national schedules. As IVIG particularly supresses the response to measles vaccine, and in line with recent AHA guidance, we also suggest that MMR vaccine (and VZV vaccine, albeit with less supporting evidence) might be deferred for at least 11 months after IVIG administration[3]. We however acknowledge that children at high-risk of exposure to measles should be vaccinated earlier than this 11-month window, with the possibility of re-vaccination if serological response is suboptimal.

\section{Management of other KD scenarios}

A wide range of other complex management scenarios arising in the care of children with $K D$, spanning acute scenarios (such as emergency thrombolysis, calculation of coronary Z scores, long-term follow-up strategies and transition to adult care) were beyond the scope of the SHARE process but are covered elsewhere.[3,5]

\section{Conclusions}

The SHARE recommendations provide international, evidence-based consensus recommendations for the diagnosis and treatment of $K D$ in children, facilitating improvement and uniformity of care. A total of 17 recommendations for diagnosis, and 14 for treatment were accepted with $100 \%$ agreement. In developing these recommendations, the importance of ongoing and future clinical trials/studies in KD was recognised to further improve the diagnosis, treatment, and monitoring into adulthood of these patients. 


\section{Acknowledgements}

We would like to thank all those who contributed to the SHARE initiative, and especially their suggestions, advice and expertise to the paediatric systemic vasculitis workstream.

\section{Contributors}

NW and BV designed the SHARE initiative. NdG and NG performed the systematic literature review, supervised by MWB and SK. Validity assessment of selected papers was done by SO, PB, LM, AvR, DE and MT. Recommendations were formulated by NdG, NG, SK, PB, and MWB. The expert committee consisted of PB, SO, TA, BB-M, PD, IK-P, PL, SDM, LM, CP, AvR, YU, NW, SK and MWB; they completed the online surveys and/or participated in the subsequent consensus meetings. NdG, NG, SK and MWB prepared, and NdG and NG chaired the consensus meetings and took minutes. $\mathrm{AR}$ and $\mathrm{BF}$ facilitated the consensus procedure using nominal group technique. NdG, DE, SK, PB and MWB wrote the manuscript, with contribution and approval of all co-authors. PB supported and MWB oversaw all aspects as senior authors. 


\section{References}

1. Gardner-Medwin JM, Dolezalova P, Cummins C, Southwood TR Incidence of HenochSchonlein purpura, Kawasaki disease, and rare vasculitides in children of different ethnic origins. Lancet 360; 1197-202.

2. Makino N, Nakamura $\mathrm{Y}$, Yashiro $\mathrm{M}$ et al. Epidemiological observations of Kawasaki disease in Japan, 2013-2014. Pediatr Int.

3. McCrindle BW, Rowley AH, Newburger JW et al. Diagnosis, Treatment, and LongTerm Management of Kawasaki Disease: A Scientific Statement for Health Professionals From the American Heart Association. Circulation 135; e927-e999.

4. Tulloh RM, Ramanan AV, Brogan P et al. Kawasaki disease. Results of the BPSU survey in UK and Ireland. Archives of disease in childhood 101; A5.

5. Eleftheriou D, Levin M, Shingadia D, Tulloh R, Klein NJ, Brogan PA Management of Kawasaki disease. Arch Dis Child 99; 74-83.

6. Shah V, Christov G, Mukasa T et al. Cardiovascular status after Kawasaki disease in the UK. Heart 101; 1646-55.

7. Suda $\mathrm{K}$, lemura $\mathrm{M}$, Nishiono $\mathrm{H}$ et al. Long-term prognosis of patients with Kawasaki disease complicated by giant coronary aneurysms: a single-institution experience. Circulation 123; 1836-42.

8. Wulffraat NM, Vastert B Time to share. Pediatr Rheumatol Online J 11; 5.

9. Enders FB, Bader-Meunier B, Baildam E et al. Consensus-based recommendations for the management of juvenile dermatomyositis. Ann Rheum Dis.

10. Giancane G, Ter Haar NM, Wulffraat $N$ et al. Evidence-based recommendations for genetic diagnosis of familial Mediterranean fever. Ann Rheum Dis 74; 635-41.

11. Groot N, de GN, Avcin $T$ et al. European evidence-based recommendations for diagnosis and treatment of paediatric antiphospholipid syndrome: the SHARE initiative. Ann Rheum Dis.

12. Groot N, Graeff N, Avcin T et al. European evidence-based recommendations for diagnosis and treatment of childhood-onset systemic lupus erythematosus: the SHARE initiative. Ann Rheum Dis.

13. Ter Haar NM, Oswald M, Jeyaratnam $\mathrm{J}$ et al. Recommendations for the management of autoinflammatory diseases. Ann Rheum Dis 74; 1636-44.

14. Newburger JW, Takahashi M, Gerber MA et al. Diagnosis, treatment, and long-term management of Kawasaki disease: a statement for health professionals from the Committee on Rheumatic Fever, Endocarditis and Kawasaki Disease, Council on Cardiovascular Disease in the Young, American Heart Association. Circulation 110; 2747-71.

15. Dougados M, Betteridge N, Burmester GR et al. EULAR standardised operating procedures for the elaboration, evaluation, dissemination, and implementation of recommendations endorsed by the EULAR standing committees. Ann Rheum Dis 63; $1172-6$

16. Leclercq E, Leeflang MM, van Dalen EC, Kremer LC Validation of search filters for identifying pediatric studies in PubMed. J Pediatr 162; 629-34.

17. Whiting P, Rutjes AW, Reitsma JB, Glas AS, Bossuyt PM, Kleijnen J Sources of variation and bias in studies of diagnostic accuracy: a systematic review. Ann Intern Med 140; 189-202.

18. Higgins JPT, Green S, The Cochrane Collaboration 2 Cochrane Handbook for Systematic Reviews of Interventions Version 5.1.0. http://handbook.cochrane.org. Online resource.

19. Zhang W, Doherty M, Pascual E et al. EULAR evidence based recommendations for gout. Part I: Diagnosis. Report of a task force of the Standing Committee for International Clinical Studies Including Therapeutics (ESCISIT). Ann Rheum Dis 65; 1301-11.

20. Zhang W, Doherty M, Bardin T et al. EULAR evidence based recommendations for gout. Part II: Management. Report of a task force of the EULAR Standing Committee for International Clinical Studies Including Therapeutics (ESCISIT). Ann Rheum Dis 65; 1312-24.

21. Delbecq AL, Van de Ven A A group process model for problem identification and program planning. The Journal of Applied Behavioural Science 7; 466-92. 
22. Brogan PA, Bose A, Burgner D et al. Kawasaki disease: an evidence based approach to diagnosis, treatment, and proposals for future research. Arch Dis Child 86; 286-90.

23. Lee KY, Lee HS, Hong JH, Han JW, Lee JS, Whang KT High-dose intravenous immunoglobulin downregulates the activated levels of inflammatory indices except erythrocyte sedimentation rate in acute stage of Kawasaki Disease. J Trop Pediatr 51; 98-101.

24. Salehzadeh F, Noshin A, Jahangiri S IVIG Effects on Erythrocyte Sedimentation Rate in Children. Int J Pediatr 2014; 981465.

25. Benseler SM, McCrindle BW, Silverman ED, Tyrrell PN, Wong J, Yeung RS Infections and Kawasaki disease: implications for coronary artery outcome. Pediatrics 116; e760e766.

26. Egami $\mathrm{K}$, Muta $\mathrm{H}$, Ishii $\mathrm{M}$ et al. Prediction of resistance to intravenous immunoglobulin treatment in patients with Kawasaki disease. J Pediatr 149; 237-40.

27. Kobayashi $\mathrm{T}$, Inoue $\mathrm{Y}$, Takeuchi $\mathrm{K}$ et al. Prediction of intravenous immunoglobulin unresponsiveness in patients with Kawasaki disease. Circulation 113; 2606-12.

28. Sano T, Kurotobi S, Matsuzaki K et al. Prediction of non-responsiveness to standard high-dose gamma-globulin therapy in patients with acute Kawasaki disease before starting initial treatment. Eur J Pediatr.

29. Sleeper LA, Minich LL, McCrindle BM et al. Evaluation of Kawasaki disease riskscoring systems for intravenous immunoglobulin resistance. J Pediatr 158; 831-5.

30. Davies S, Sutton N, Blackstock $S$ et al. Predicting IVIG resistance in UK Kawasaki disease. Arch Dis Child 100; 366-8.

31. Jakob A, von KR, Horstmann J et al. Failure to Predict High-Risk Kawasaki Disease Patients in a Population-Based Study Cohort in Germany. Pediatr Infect Dis J.

32. Dallaire F, Fournier A, Breton J, Nguyen TD, Spigelblatt L, Dahdah N Marked variations in serial coronary artery diameter measures in Kawasaki disease: a new indicator of coronary involvement. J Am Soc Echocardiogr 25; 859-65.

33. Manlhiot C, Millar K, Golding F, McCrindle BW Improved classification of coronary artery abnormalities based only on coronary artery z-scores after Kawasaki disease. Pediatr Cardiol 31; 242-9.

34. McCrindle BW, Li JS, Minich LL et al. Coronary artery involvement in children with Kawasaki disease: risk factors from analysis of serial normalized measurements. Circulation 116; 174-9.

35. Ogata $\mathrm{S}$, Tremoulet $\mathrm{AH}$, Sato $\mathrm{Y}$ et al. Coronary artery outcomes among children with Kawasaki disease in the United States and Japan. Int J Cardiol 168; 3825-8.

36. Kobayashi T, Saji T, Otani T et al. Efficacy of immunoglobulin plus prednisolone for prevention of coronary artery abnormalities in severe Kawasaki disease (RAISE study): a randomised, open-label, blinded-endpoints trial. Lancet 379; 1613-20.

37. Binstadt BA, Levine JC, Nigrovic PA et al. Coronary artery dilation among patients presenting with systemic-onset juvenile idiopathic arthritis. Pediatrics 116; e89-e93.

38. Durongpisitkul K, Gururaj VJ, Park JM, Martin CF The prevention of coronary artery aneurysm in Kawasaki disease: a meta-analysis on the efficacy of aspirin and immunoglobulin treatment. Pediatrics 96; 1057-61.

39. Newburger JW, Takahashi M, Burns JC et al. The treatment of Kawasaki syndrome with intravenous gamma globulin. N Engl J Med 315; 341-7.

40. Newburger JW, Takahashi M, Beiser AS et al. A single intravenous infusion of gamma globulin as compared with four infusions in the treatment of acute Kawasaki syndrome. N Engl J Med 324; 1633-9.

41. Terai M, Shulman ST Prevalence of coronary artery abnormalities in Kawasaki disease is highly dependent on gamma globulin dose but independent of salicylate dose. $J$ Pediatr 131; 888-93.

42. Amarilyo G, Koren Y, Brik SD et al. High-dose aspirin for Kawasaki disease: outdated myth or effective aid? Clin Exp Rheumatol 35 Suppl 103; 209-12.

43. Chen S, Dong Y, Yin Y, Krucoff MW Intravenous immunoglobulin plus corticosteroid to prevent coronary artery abnormalities in Kawasaki disease: a meta-analysis. Heart 99; 76-82.

44. Chen S, Dong Y, Kiuchi MG et al. Coronary Artery Complication in Kawasaki Disease and the Importance of Early Intervention : A Systematic Review and Meta-analysis. JAMA Pediatr. 
45. Tremoulet $\mathrm{AH}$, Best BM, Song $\mathrm{S}$ et al. Resistance to intravenous immunoglobulin in children with Kawasaki disease. J Pediatr 153; 117-21.

46. Dhillon R, Newton L, Rudd PT, Hall SM Management of Kawasaki disease in the British Isles. Arch Dis Child 69; 631-6.

47. Lyskina G, Bockeria O, Shirinsky O et al. Cardiovascular outcomes following Kawasaki disease in Moscow, Russia: A single center experience. Glob Cardiol Sci Pract 2017; e201723.

48. Mossberg M, Segelmark M, Kahn R, Englund M, Mohammad AJ Epidemiology of primary systemic vasculitis in children: a population-based study from southern Sweden. Scand J Rheumatol 1-8.

49. Brogan $P$, Levin $M$ Intravenous immunoglobulin plus corticosteroids prevent coronary artery abnormalities in Kawasaki disease. Evid Based Med 18; 217-8.

50. Furukawa T, Kishiro M, Akimoto K, Nagata S, Shimizu T, Yamashiro Y Effects of steroid pulse therapy on immunoglobulin-resistant Kawasaki disease. Arch Dis Child 93; $142-$ 6.

51. Inoue $\mathrm{Y}$, Okada $\mathrm{Y}$, Shinohara $\mathrm{M}$ et al. A multicenter prospective randomized trial of corticosteroids in primary therapy for Kawasaki disease: clinical course and coronary artery outcome. J Pediatr 149; 336-41.

52. Okada K, Hara J, Maki I et al. Pulse methylprednisolone with gammaglobulin as an initial treatment for acute Kawasaki disease. Eur J Pediatr 168; 181-5.

53. Sundel RP, Baker AL, Fulton DR, Newburger JW Corticosteroids in the initial treatment of Kawasaki disease: report of a randomized trial. J Pediatr 142; 611-6.

54. Latino GA, Manlhiot C, Yeung RS, Chahal N, McCrindle BW Macrophage Activation Syndrome in the Acute Phase of Kawasaki Disease. J Pediatr Hematol Oncol.

55. Dominguez SR, Friedman K, Seewald R, Anderson MS, Willis L, Glode MP Kawasaki disease in a pediatric intensive care unit: a case-control study. Pediatrics 122; e786e790.

56. Kanegaye JT, Wilder MS, Molkara D et al. Recognition of a Kawasaki disease shock syndrome. Pediatrics 123; e783-e789.

57. Burns JC, Mason WH, Hauger SB et al. Infliximab treatment for refractory Kawasaki syndrome. J Pediatr 146; 662-7.

58. Burns JC, Best BM, Mejias A et al. Infliximab treatment of intravenous immunoglobulinresistant Kawasaki disease. J Pediatr 153; 833-8.

59. Choueiter NF, Olson AK, Shen DD, Portman MA Prospective open-label trial of etanercept as adjunctive therapy for kawasaki disease. J Pediatr 157; 960-6.

60. Son MB, Gauvreau K, Burns JC et al. Infliximab for intravenous immunoglobulin resistance in Kawasaki disease: a retrospective study. J Pediatr 158; 644-9.

61. Tremoulet $\mathrm{AH}$, Jain $\mathrm{S}$, Jaggi $\mathrm{P}$ et al. Infliximab for intensification of primary therapy for Kawasaki disease: a phase 3 randomised, double-blind, placebo-controlled trial. The Lancet 383; 1731-8.

62. Sugahara $\mathrm{Y}$, Ishii $\mathrm{M}$, Muta $\mathrm{H}$, lemura $\mathrm{M}$, Matsuishi $\mathrm{T}$, Kato $\mathrm{H}$ Warfarin therapy for giant aneurysm prevents myocardial infarction in Kawasaki disease. Pediatr Cardiol 29; 398401.

63. Weiss $P$, Soff $G A$, Halkin $H$, Seligsohn $U$ Decline of proteins $C$ and $S$ and factors II, VII, IX and X during the initiation of warfarin therapy. Thromb Res 45; 783-90.

64. Esposito S, Bianchini S, Dellepiane RM, Principi N Vaccines and Kawasaki disease. Expert Rev Vaccines 15; 417-24.

65. Looney RJ, Huggins J Use of intravenous immunoglobulin G (IVIG). Best Pract Res Clin Haematol 19; 3-25. 